\title{
Revisiting the dedifferentiation hypothesis with longitudinal multi-cohort data
}

\author{
Cindy M. de Frias ${ }^{\mathrm{a}, *}$, Martin Lövdén ${ }^{\mathrm{b}}$, Ulman Lindenberger ${ }^{\mathrm{b}}$, Lars-Göran Nilsson ${ }^{\mathrm{c}}$ \\ ${ }^{a}$ Division of Neurology, University of Alberta, Edmonton, Alberta, Canada \\ ${ }^{\mathrm{b}}$ Center for Lifespan Psychology, Max Planck Institute for Human Development, Berlin, Germany \\ ${ }^{\mathrm{c}}$ Stockholm University, Stockholm, Sweden
}

Received 9 March 2006; received in revised form 12 July 2006; accepted 13 July 2006

Available online 28 November 2006

\begin{abstract}
The present longitudinal multi-cohort study examines whether interindividual variability in cognitive performance and change increases in old age, and whether associations among developments of different cognitive functions increase with adult age. Multivariate multiple-group latent growth modeling was applied to data from narrow cohorts separated by five years of age. Tests assessing episodic recall, semantic knowledge, semantic fluency, and visuospatial ability were administered to 1000 non-demented adults (initially aged 35-80 years), participating in the Betula Project at three occasions over a 10-year period. Greater interindividual differences in change were noted in older age groups. Age-related increases in correlations among performance scores were noted for different cognitive measures beginning in old age, but not earlier. Our study supports a dynamic view of dedifferentiation of cognitive aging.
\end{abstract}

(C) 2006 Elsevier Inc. All rights reserved.

Keywords: Dedifferentiation hypothesis; Aging; Multi-cohort design

Age-comparative analyses of the relations between changes in multiple cognitive domains are almost absent from the developmental literature (for review, see Baltes, Lindenberger, \& Staudinger, 2006). Particularly with respect to intellectual development, previous studies have generally examined cognitive changes within a single cohort. In contrast, the present report compares the magnitude and structure (variances and correlations) of cognitive change across different age cohorts (see

\footnotetext{
* Corresponding author.

E-mail addresses: defrias@ualberta.ca (C.M. de Frias), loevden@mpib-berlin.mpg.de (M. Lövdén), lindenberger@mpib-berlin.mpg.de (U. Lindenberger), lgn@psychology.su.se (L.-G. Nilsson).
}

also Anstey, Hofer, \& Luszcz, 2003a). Our two main predictions are that (a) there are increasing interindividual differences with age in rates of change in cognitive abilities and (b) how individuals change in one cognitive ability is increasingly related to the ways they change in other cognitive abilities with advancing age. To test these predictions, we applied multivariate, multi-cohort latent growth modeling (LGM) to three-occasion, tenyear longitudinal data covering four cognitive abilities.

Prior research has reported interindividual differences in cognitive changes in midlife to old age (Hultsch, Hertzog, Dixon, \& Small, 1998; Lövdén et al., 2004; Wilson et al., 2002). We examined whether interindividual differences in different cognitive abilities increase with age because interindividual differences in change 
are a prerequisite for testing the second hypothesis. If rearrangement of rank-ordering does not occur, then correlations do not change.

An important question in cognitive aging research concerns age differences in the multivariate structure, or dimensionality, of cognitive changes (e.g., Allen et al., 2001; Hofer \& Sliwinski, 2001; Lindenberger \& Baltes, 1994; Rabbitt, 1993). Here, the crucial task is to assess age differences in the degree of commonality among changes in different cognitive abilities; that is, the degree to which changes in a single cognitive ability are associated with changes in other abilities. Two strands of research provide key empirical evidence on this topic. First, results from several longitudinal studies indicate that correlations among changes in different cognitive abilities are of moderate to high magnitude in samples of older individuals (Anstey, Hofer, \& Luszcz, 2003b; Hertzog, Dixon, Hultsch, \& MacDonald, 2003; Hultsch et al, 1998; Lövdén et al., 2004; Wilson et al., 2002; but see Lövdén, Bergman, Adolfsson, Lindenberger, \& Nilsson, 2005; Sliwinski, Hofer, \& Hall, 2003). These substantial correlations indicate commonality in rates of change, but the lack of unity indicates unique changes across cognitive functions in old age. Second, evidence of dedifferentiation, that is, an increase in correlations between levels of cognitive performances as a function of adult age (i.e., Balinsky, 1941; see also Baltes \& Lindenberger, 1997; Ghisletta \& Lindenberger, 2003; Hofer \& Sliwinski, 2001; Li et al., 2004; Li \& Schmiedek, 2002), indicates correlated developmental changes. Specifically, given that interindividual differences in cognitive aging are present, rank ordering of individuals' performance levels should be more strongly determined by aging-related variance in samples of older adults than in samples of younger adults. Accordingly, if age-related changes in different abilities are associated, the correlation among levels of performance on different abilities should increase as a function of age. In other words, comparing correlations among cognitive performance levels across age groups indirectly speaks to whether age-related changes in different cognitive abilities are associated (Hofer \& Sliwinski, 2001).

Two types of dedifferentiation can be distinguished: dynamic and stationary dedifferentiation (Lövdén \& Lindenberger, 2005). The notion that an ensemble of common sources increasingly dominates development of intellectual abilities is a cornerstone of the dynamic dedifferentiation hypothesis. Such sources might point to normative and generalized neuroanatomical and neurochemical decline occurring in, but not before, old age (Raz et al., 2005). Dynamic dedifferentiation may also be observed when increasing proportions of individuals are afflicted by impairments separable from normal aging such as terminal decline (e.g., Lövdén \& Lindenberger, 2005; Singer, Verhaeghen, Ghisletta, Lindenberger, \& Baltes, 2003) and dementia (Sliwinski et al., 2003).

Distinguishing between the dynamic view and the stationary view will inform cognitive aging theories about the generalizability of biological mechanisms that explain within-person changes in cognitive functioning in younger and later adulthood. Interindividual differences in cognitive change should be higher in very late life, at a time when non-normative sources of heterogeneity (e.g., preclinical dementia, terminal decline) override normative age-related change. Because interindividual differences in change are a prerequisite for dedifferentiation at the level of interindividual differences, age-related or non-linear increases in variances would be in line with the dynamic dedifferentiation hypothesis (especially if variances in change are very small to non-existent in samples of younger adults). ${ }^{1}$ To emphasize, if interindividual differences in change are very small or non-existent in groups of younger adults, then higher correlations among cognitive abilities as a function of advancing age cannot occur in younger adulthood. The stationary view of dedifferentiation (similar associations among changes in old age as in younger adulthood) would be represented by age-invariant correlations among changes in different cognitive abilities and by linear increases in correlations among levels of performances on different abilities.

The evidence for age-related dedifferentiation examined through age-related differences in correlations among levels of performances in different abilities is inconclusive. Whereas some studies have found age differences in correlations between cognitive variables (e.g., Baltes \& Lindenberger, 1997; Hultsch et al., 1998), others have found no change in the correlations among manifest variables (Zelinski \& Stewart, 1998) or latent variables (Zelinski \& Lewis, 2003) with age. Furthermore, using 3-year longitudinal data, Zelinski and Lewis (2003) found no structural differences (i.e., invariance in factor loadings, covariances, and variances) on latent cognitive variables. Similarly, Anstey et al. (2003a) failed to find consistent patterns of longitudinal dedifferentiation. Schaie, Maitland, Willis, \& Intrieri (1998) found a seven-year longitudinal increase in the covariances only for the oldest-old.

\footnotetext{
${ }^{1}$ Because of the non-linear properties of the Pearson correlation, even a linear increase would in fact support the dynamic dedifferentiatin hypothesis. However, linearity constitutes a straightforward and rigorous null hypothesis.
} 
In this study, we made use of three-wave (10-year) longitudinal data from the Betula Project (Nilsson et al., 2004). At first assessment, this study contained a sample of 1,000 participants, 100 in each of ten cohorts (35, $40, \ldots$, and 80 years old). Thus, the study includes the desirable feature of having sampled sequential narrow cohorts. Specifically, covariances among age-related variables in cross-sectional samples are influenced by average age-related differences, initial individual differences, and individual differences in rates of aging. An alternative approach to age-heterogenous crosssectional and longitudinal designs is the sequential narrow-age cohort (SNAC) design that consists of sequential cohorts of individuals of nearly the same chronological age (Hofer \& Sliwinski, 2001; Hofer, Sliwinski, \& Flaherty, 2002). To the extent that aging processes are captured by differences and changes in chronological age, the SNAC design allows for rigorous examination of differences in covariances across different age groups because the mean trends are not confounding the covariances. Specifically, if the groups were age-heterogeneous, correlations among cognitive abilities would be inflated owing to the common association with age. In the present context, the SNAC design is also advantageous because it allows a finegrained examination of almost continuous differences in correlations and variances across the adult lifespan.

Utilizing cross-sectional data from the Betula study, Nyberg et al. (2003) demonstrated structural stability of declarative memory in young and old adults and also reported that a four-factor model solution had a better fit than a two-factor model solution involving measures of episodic memory (recall and recognition) and semantic memory (fluency and knowledge). Configural (same pattern of factor loadings) and metric (same magnitude of factor loadings) invariance over age for this model has been supported in both cross-sectional (Nyberg et al., 2003) and longitudinal (Lövdén et al., 2005; Lövdén et al., 2004) data. In this study, we examine variances and covariances among measures of recall, fluency, and knowledge. The selection of specific measures of these constructs were based on satisfactory psychometric properties (e.g., reliability, distribution) and longitudinal availability of the measures (cf. Rönnlund, Nyberg, Bäckman, \& Nilsson, 2005). In addition, we included a marker of visuo-spatial ability (Block Design).

Further recent longitudinal investigations from the Betula Study reported 5-year mean-level stability of episodic memory up to age 60 years, and improvement of semantic memory up to 55 years of age after controlling for practice effects (Rönnlund et al., 2005). The differ- ential aging patterns suggest that multiple sources may affect these factors, or that the factors have a different association to a common age-related factor. Tentative support for a small increase in the correlation between episodic and semantic memory in a short (5-year) measurement interval has been reported in adults aged 60 to 80 years (Lövdén et al., 2005), suggesting that dedifferentiation of declarative memory may reside at some point after midlife.

In line with the findings of Schaie et al., and in line with the dynamic dedifferentiation hypothesis, we expected no or weak patterns of dedifferentiation before old and very old age. Specifically, based on findings suggesting that terminal decline and preclinical dementia is associated with general declines across abilities (e.g., Lövdén et al., 2005; Singer et al., 2003; Sliwinski et al., 2003) and given that these sources of cognitive aging increase exponentially over age, we expected stronger associations among cognitive changes in older than in younger adulthood. We assumed that cognitive skill levels would essentially merge in very old age, and previous divisions between relative strengths and weaknesses in abilities would eventually dissolve.

We analyzed four cognitive measures with a multivariate multi-cohort (i.e., age-group) LGM. Theoretical and methodological features of the selected cognitive constructs make them valid for addressing the dedifferentiation hypothesis: (a) They have differential longitudinal trajectories (i.e., stability of episodic memory up to age 60 years, and improvement of semantic memory into midlife), and (b) construct validity has been previously documented (i.e., four distinct declarative memory subsystems). With the LGM approach, we can produce the estimates of interest for this study: (a) interindividual differences in changes of performance as a function of age group (i.e., age differences in slope variances), (b) correlation among changes in performance for different cognitive abilities as a function of age group (i.e., age differences in correlation among slopes), and (c) correlations among levels of performance as a function of age groups (i.e., age differences in intercept correlations). In line with the dynamic dedifferentiation hypothesis, we predicted increasing interindividual differences and increasing correlations in, but not before, old age.

\section{Methods}

\subsection{Participants}

Participants were drawn from Sample 1 (S1) of the Betula Project (Nilsson et al., 1997, 2004). All participants of the Betula Project were drawn from the 
population registry in Umeå, a city in northern Sweden, with 100,000 inhabitants. Participants were tested over a 10-year (3-wave) period at 5-year intervals (Time 1: T1 1988-1990; Time 2: T2 1993-1995; Time 3: T3 19982000). Participants were screened for dementia before inclusion into the study.

At T1, the sample consisted of 1000 participants, 100 in each of ten cohorts (35-, 40-,..., and 80-year-olds). At $\mathrm{T} 2$, the sample was reduced to 850 participants. Of those who had dropped out at T2, 52 participants were deceased, 28 were demented, and 70 had dropped out for experimental reasons (i.e., they refused to participate, moved, were ill, not available, or had missing values on all variables central to this study). At T3, the sample consisted of 649 individuals. Of those who had dropped out at T3, 166 participants were deceased, 49 were demented, and 136 had dropped out for experimental reasons. The way this dropout is handled in the analysis is outlined in the Statistical Procedures. For this study, we partitioned the sample into five age groups, 35-40, $45-50,55-60,65-70$, and $75-80$ years old at T1.

After a complete description of the study to the participants, written informed consent was obtained.

\subsection{Procedure and measures}

At all three occasions, the measures were obtained during two test sessions, each lasting for 1.5 to 2 hours for each participant. The first session consisted of health examinations and questionnaires. The second session included an extensive battery of cognitive tasks. We examine measures of recall ( 3 tasks), fluency ( 3 tasks), knowledge (1 task), and visuospatial ability (1 task). By standardizing each individual measure, summing the measures for each factor separately, and standardizing the sums to the T-metric, we formed four unit-weighted composites to use in the analyses. The 35- to 40-yearolds' $M s$ and SDs at T1 provided the reference for all standardizations.

\subsubsection{Block design}

The obtained raw scores from the WAIS Block Design test (Wechsler, 1991) were used to measure visuospatial ability. In this speeded task, participants were required to place red and white blocks such that they formed the same pattern shown on a target paper. The ten-year test-retest correlation of this measure was .79 for the youngest group, indicating a satisfactory lower reliability boundary.

\subsubsection{Episodic recall}

In the study phase, each participant was presented with two consecutive lists of imperatives (e.g., roll the ball) presented at the rate of $8 \mathrm{~s} /$ item. The nouns in the sentences were derived from eight semantic categories, with four items in each category. During the study phase of one of the lists, participants were requested to perform the action described by the imperatives, using an object corresponding to the noun (enacted condition). The first measure of recall was a free recall test that followed after each list, and the performance score was the number of sentences (correct verb and noun) recalled in the enacted condition. After the free recall test, participants were given the eight category names, which were used as cues to remember the nouns. The second and third measures of recall were the number of nouns (cued)recalled from the enacted and non-enacted conditions, respectively. The ten-year test-retest correlation of the composite of these three measures was .67 for the youngest group.

\subsubsection{Semantic fluency}

Three fluency tasks were administered in which the participants were instructed to generate aloud as many words as possible in one minute. The differences in the tasks concerned the category of words to be generated. The first task was to produce words beginning with the letter A. The second task required generation of words beginning with $\mathrm{M}$ and containing five letters. The third task was to produce professions beginning with the letter B. The ten-year test-retest correlation of the composite was .65 for the youngest group.

\subsubsection{Semantic knowledge}

The knowledge measure was derived from a 30-item multiple-choice synonym test (Dureman, 1960). Participants selected a synonym for each target word from five alternatives. The total number of correctly selected synonyms in seven minutes yielded a measure of verbal knowledge. The ten-year test-retest correlation of this measure was .79 for the youngest group.

\subsection{Statistical procedures}

We analyzed the data with structural equation modeling and, specifically, with multivariate multi-cohort LGM. A univariate LGM (McArdle \& Epstein, 1987; Meredith \& Tisak, 1990; Raudenbush \& Bryk, 2002) proposes two latent variables, an intercept and a slope, to account for the longitudinal time series information. In this application, the loading of the observed measure on the slope factor was set to 0 at $\mathrm{T} 1$ and the loading at T3 was set to 10 to capture the ten-year study period. Thus, the intercept represents an individual's latent score at the beginning of the time series (i.e., at T1) and 




Fig. 1. Multivariate (Block Design, verbal fluency, recall, and verbal knowledge) latent growth model as implemented here. Unlabeled paths are fixed to 1. Covariances are not shown for space reasons, but we attempted to estimate all intercept-intercept, slope-slope, and intercept-slope covariances. The depicted model was estimated as a multi-group model (35- to 40-, 45- to 50-, 55- to 60-, 65- to 70-, and 75- to 80-year-old groups). BD = Block Design; FLU = verbal fluency; $\mathrm{RC}=$ recall; $\mathrm{KNO}=$ verbal knowledge; $\mathrm{S}=$ slope; $\mathrm{IC}=$ intercept; Var $=$ variance; $\mathrm{SH}=$ freely estimated shape parameter.

the slope represents change scores. At T2, the loading was freely estimated so that the shape (e.g., linear, quadratic) of change could be freely estimated. Thus, the variance in the slope factor refers to deviations from the typical mean trend for the group. As compared to the more common procedure of estimating explicit polynomials, this free-basis loading approach reduces differences between groups in the fit of the average shape of change. Thus, any biases in variances of change across age groups are minimized. Both intercept and slope factors are estimated at the population level (i.e., their means are estimated), both allow for interindividual differences (i.e., their variances are estimated), and they are free to covary. The error variances are commonly assumed neither to correlate with themselves nor to change over time. Fig. 1 displays a graphical representation of the multivariate LGM implemented in this study. 
The model depicted in Fig. 1 was estimated as a multigroup model of five age groups (i.e., $35-40,45-50,55-$ $60,65-70$, and $75-80$ years old) to examine the parameter estimates as a function of age group. We employed full information maximum likelihood (FIML; Arbuckle, 1996). FIML uses all available data points and results in more exact and less biased estimates than other common ways of dealing with missing values (e.g., listwise deletion, regression imputation, mean imputation; e.g., Schafer \& Graham, 2002; Wothke, 2000). The FIML algorithm assumes missing-at-random (MAR: Rubin, 1974; see Schafer \& Graham, 2002, for a nontechnical treatment), which means that the probability that a score on $\mathrm{X}$ is missing may depend on other variables but not on $\mathrm{X}$ itself. An association between missing data and $\mathrm{X}$ stemming from the mutual association to the other variables in the model is also allowed, but no residual relationship between missing data and $\mathrm{X}$ is allowed once the other variables are taken into account. The FIML procedure provides accurate estimates when the MAR assumption is met by including satisfactory predictors of missing data in the model. In longitudinal applications like the present one, levels of performance and observed change are powerful predictors of future participation. Thus, although not directly testable, the assumption is appropriate in the context of the present study.

We utilized AMOS 5.0 (Arbuckle \& Wothke, 1999) for all computations. The alpha-level for all statistical decisions was .01 and the difference in $\chi^{2}$ fit statistics $\left(\Delta \chi^{2}\right)$ was used to compare nested models. Model fit was evaluated with the following fit indices: $\chi^{2} / d f$, Comparative Fit Index (CFI), and Root Mean Square Error of Approximation (RMSEA) (see Kline, 1998, for interpretation of these indices).

\section{Results}

We estimated the multivariate (recall, Block Design, fluency, and knowledge) LGM depicted in Fig. 1 as a multi-group (age-group) model. Several of the variances in change were negative (recall and Block Design: 35 - to 40-year-olds; recall: 45- to 50-year-olds; and recall: 55- to 60-year-olds). Because the fit of the model was acceptable, the confidence intervals of these negative estimates included 0 , and the standard errors were about the same size as those for the other estimated variances, we treated the negative sign as a value of 0 (Dillon, Kumar, \& Mulani, 1987). Thus, we fixed these variances to zero, which provided an acceptable starting model, $\chi^{2}=257.32, d f=194, p=.002, \mathrm{CFI}=.990$, RMSEA $=.018$.

\subsection{Intercept variances}

Fig. 2A shows the estimates of the intercept variances as a function of age group and cognitive variable. To address whether the intercept variances differed across age groups we specified a model assuming the variances to be equal across age groups (but allowing them to differ across measures). This model fit the data significantly worse than the starting model, $\chi^{2}=306.21, d f=210$, $p<.001$, CFI $=.985$, RMSEA $=.021 ; \Delta \chi^{2}=48.88$,
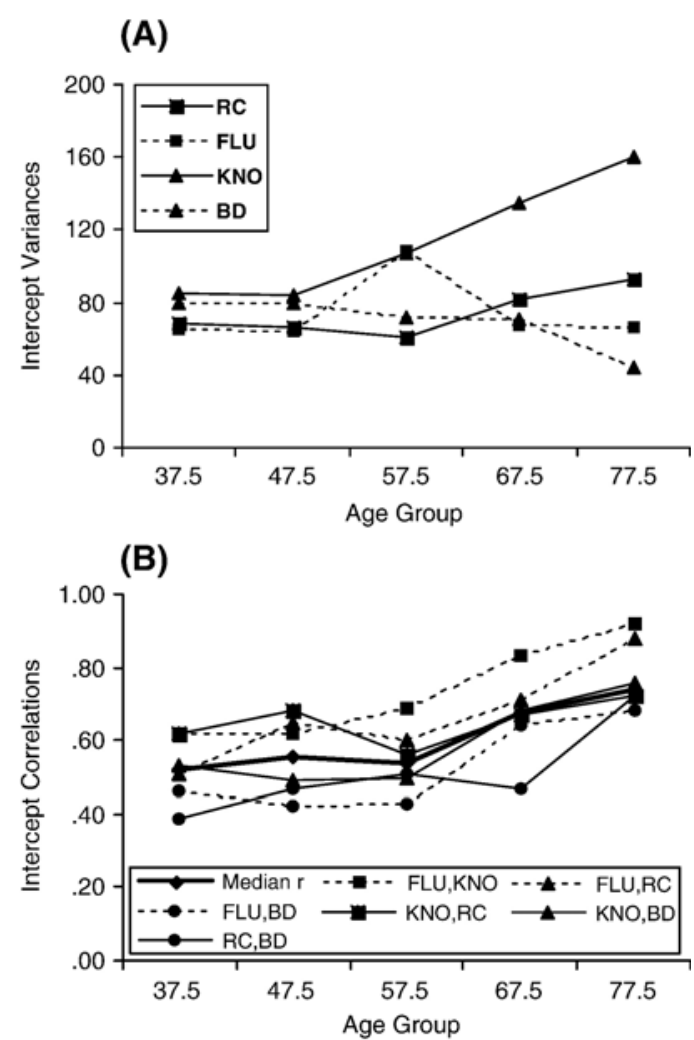

(C)

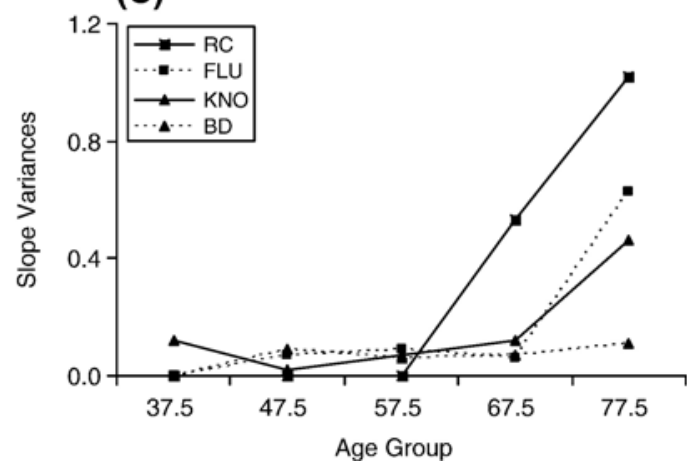

Fig. 2. Parameter estimates of (A) intercept variances, (B) intercept correlations, and $(\mathrm{C})$ slope variances as a function of age group. $\mathrm{BD}=$ Block Design; FLU = verbal fluency; $\mathrm{RC}=$ recall; $\mathrm{KNO}=$ verbal knowledge. 
$d f=16, p<.001$. We followed this omnibus test with univariate tests, by constraining the variances across age groups for one measure at a time and then comparing the fit of these models with the starting model. These tests resulted in a decrease in fit for knowledge, $\Delta \chi^{2}=14.71$, $d f=4, p<.01$, and verbal fluency, $\Delta \chi^{2}=14.96, d f=4$, $p<.01$. However, the decrease in fit for fluency was driven by higher variances for the 55- to 60-year-old group, since a model that estimated this variance but constrained the other variances across age groups did not reduce the fit, $\Delta \chi^{2}=0.06, d f=3, p>.98$. For Block Design and recall, the univariate tests did not produce any loss in fit, $\Delta \chi^{2}=7.81, d f=4, p>.09$, and $\Delta \chi^{2}=4.17, d f=4, p>.38$, respectively. To summarize, the variances in level of performance show no systematic differences across age groups for verbal fluency, recall, and Block Design, whereas they increase for knowledge.

\subsection{Intercept correlations}

Fig. 2B displays the estimates of the correlations among the intercepts as a function of age group and cognitive variable. An inspection of this figure suggests higher correlations in the 65- to 70- and 75- to 80-yearold groups relative to the younger groups, but no agerelated differences for the younger groups. To test this observation we fixed the variances in the intercepts to equality across groups and then compared a model with the covariances freely estimated against more constrained models. This procedure was used to test standardized covariances. Setting the intercept covariances to equality across the three younger age groups (35-40, 45-50, and 55-60 years old), but allowing them to differ across measures, did not produce any loss in fit, $\Delta \chi^{2}=7.39, d f=12, p>.80$. We followed this omnibus test with univariate tests, which did not reveal any significant differences, all $\Delta \chi^{2}<3.39, d f=2, p>.18$. Thus, there were no age-related differences in the correlations among the three younger groups. However, a model assuming all covariances to be equal across all groups (but differing across measures) fit significantly worse than both the model specifying equal covariances among the three younger groups, $\Delta \chi^{2}=44.79, d f=12$, $p<.001$, and the model with freely estimated covariances, $\Delta \chi^{2}=52.18, d f=24, p<.001$. We followed these omnibus tests with univariate tests of constrained covariances across all groups compared with the model freely estimating the covariances. There was a significant age-related difference in the covariances for four

( $r_{\text {fluency, knowledge, }} r_{\text {recall, fluency, }} r_{\text {Block Design, knowledge, }}$ and $\left.r_{\text {recall, knowledge }}\right)$ of the six covariances, all $\Delta \chi^{2}>$ $12.56, d f=4$, all $p \mathrm{~s}<.01$. For the other two covariances
( $r_{\text {Block Design, fluency }}$ and $\left.r_{\text {Block Design, recall }}\right)$, all $\Delta \chi^{2}<$ 6.50, $d f=4, p \mathrm{~s}>.17$. Thus, the results show higher correlations among several cognitive variables in the 65 to 70 - and 75- to 80 -year-old age groups relative to the younger age groups. Finally, we estimated a model assuming all covariances to be equal across the two oldest age groups (but differing across measures). This model fit significantly worse than the model with freely estimated covariances, $\Delta \chi^{2}=17.81, d f=6, p<.01$. Univariate tests showed that the covariance between fluency and knowledge increased from 65- to 70-year-olds and 75 - to 80 -year-olds, $\Delta \chi^{2}=10.16, d f=1, p<.01$. There were trends $(p<.05)$ for age-group differences for three other covariances $\left(r_{\text {recall, fluency }}, r_{\text {Block Design, recall, and }}\right.$ $\left.r_{\text {recall, knowledge }}\right)$, all $\Delta \chi^{2}>4.23, d f=1, p<.04$. For all other covariances, $\Delta \chi^{2}<2.23, d f=1, p>.13$.

In summary, the results show higher correlations among several cognitive variables in the 65-70 and $75-80$ year-old age groups relative to the younger age groups, but no age differences among the younger groups.

\subsection{Slope variances}

Fig. 2C depicts the estimates for the slope variances as a function of age group and cognitive variable from the starting model. The majority of the variances were non-significant, which makes statistical comparisons across age groups redundant. The only significant slope variances were for knowledge (35-40 year olds), recall (65-70 and 75-80 year olds), and fluency (75-80 year olds); all $p \mathrm{~s}<.05$. Interindividual differences in change tend to increase in old age, but could not be detected for the majority of the measures and age groups.

\subsection{Slope correlations}

We do not report the results for the correlations among the slopes because the only empirically identified correlation was between fluency and recall in the 75 - to 80 -year-olds. That is, this correlation was the only one that could be estimated. All other slope-slope combinations involved variables displaying non-significant variances in change, thus, they were constants.

\subsection{Means}

Fig. 3 displays the implied means as a function of age group for Block Design (Fig. 3A), recall (Fig. 3B), verbal fluency (Fig. 3C), and verbal knowledge (Fig. 3D) as estimated with the starting model. The means provide information on the estimated mean shape of change and 
(A)

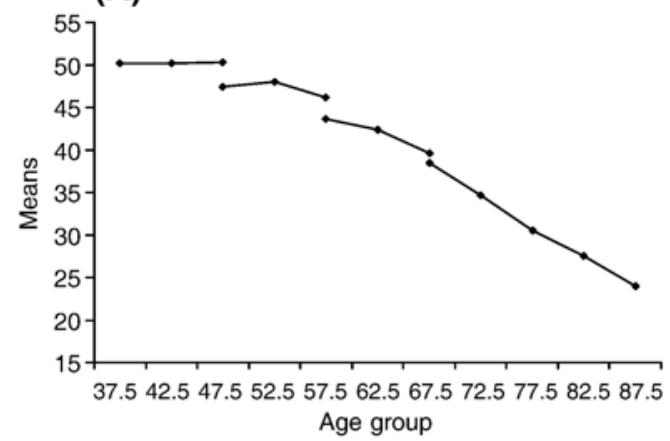

(C)

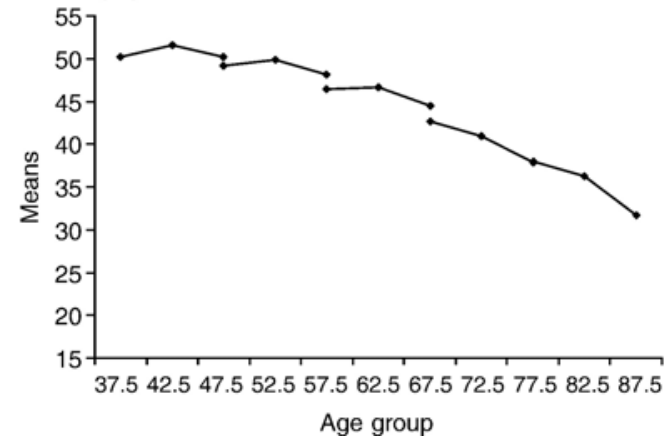

(B)

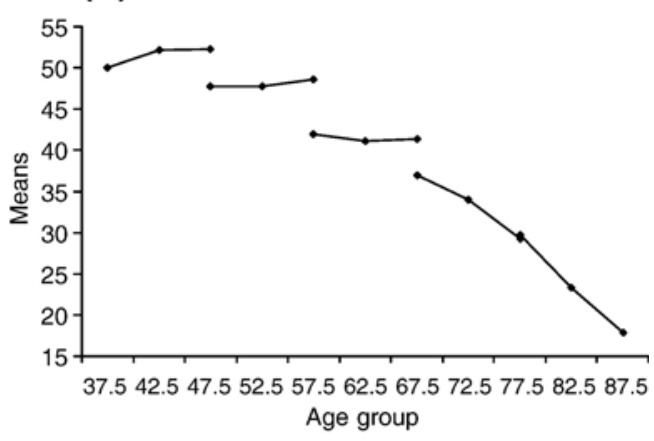

(D)

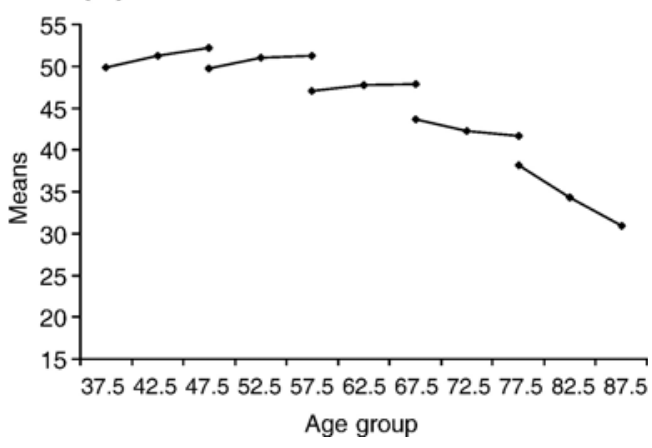

Fig. 3. Model implied means as a function of age group and time for (A) Block Design, (B) recall, (C) verbal fluency, and (D) verbal knowledge.

the magnitude of change for each of the age groups and measures. Especially relevant in this context, longitudinal average decline is greater in the older age groups than in the younger age groups. Though this pattern appears less pronounced for the initial cross-sectional differences, the mean age trends might be more pronounced in the old age groups. Thus, there is a slight possibility that the mean trends could contribute to the age-related increase in the correlations among the intercepts (i.e., the groups are not perfectly age homogenous), although each of the groups includes individuals within the same age range. We performed a control analysis, statistically controlling for chronological age by regressing the intercept factors on age, and examining the pattern of correlations among the intercepts as a function of age. This analysis revealed a substantively identical pattern of findings as those reported above (see Intercept Correlations).

\subsection{Effects of dementia and death}

To address the possibility that dementia or death during the 10-year course of the study may have influenced the findings, these cases (dementia: $n=49$; died: $n=117)$ were removed from the total sample $(N=$ 1000). We re-estimated the multivariate LGM depicted in Fig. 1 as a multi-group model, with the reduced sample $(n=785)$ providing the data. Several of the variances in change were negative (recall and Block Design: 35- to 40-year-olds; recall: 45- to 50-year-olds; recall: 55- to 60-year-olds; recall and fluency: 65- to 70year-olds; and knowledge: 75- to 80-year-olds). Again, we regarded the negative signs as indicating true estimates close to zero (Dillon et al., 1987) and fixed these variances to zero, which resulted in an acceptable starting model, $\chi^{2}=223.63, d f=197, p=.094, \mathrm{CFI}=.995$, $\mathrm{RMSEA}=.013$. Fig. 4 displays the estimates of the intercept variances (Fig. 4A), intercept correlations (Fig. 4B), and the slope variances (Fig. 4C) from this model as a function of age group. In general, these figures reveal patterns of findings that are very similar to those emanating from the analyses of the total sample (Fig. 2A-C). Specifically, (a) the intercept variances show age-related increases for knowledge only, (b) the correlations tend to be higher in the two oldest age groups for most of the six correlations, and (c) the variances in slopes tend to increase in the oldest age group as compared to the younger age groups.

We estimated models to examine whether the estimates from the model based on the reduced sample (i.e., excluding participants with impending death or dementia) differed from the corresponding estimates for 



(C)

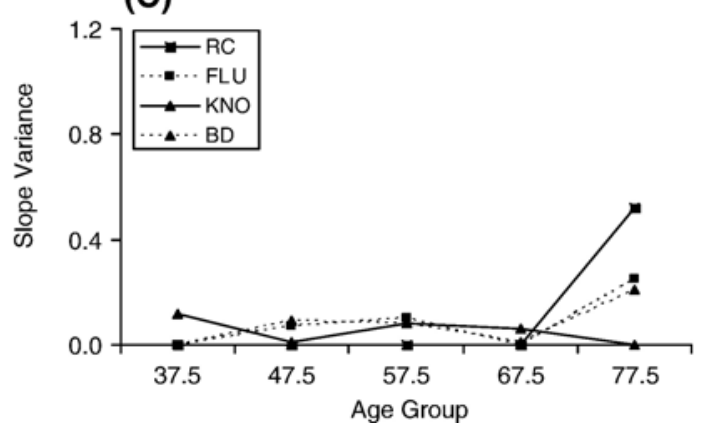

Fig. 4. Parameter estimates of (A) intercept variances, (B) the intercept correlations, (C) slope variances as a function of age group (excludes participants with impending dementia or death).

the total sample. A model, estimated with the data from the reduced sample, and assuming the intercept variances to be equal to the variances for the total sample, did not fit the data significantly worse than the starting model, $\chi^{2}=228.10, d f=217, p=.289, \mathrm{CFI}=.998, \mathrm{RMSEA}=$ $.008 ; \Delta \chi^{2}=4.48, d f=20, p>.99$. To examine potential differences in the covariances, we first fixed the variances in the intercepts to equality across groups and then compared a model with the covariances freely estimated against more constrained models. Feeding the model with the estimates of the covariances from the total sample did not reduce the fit relative to the model freely estimating the covariances, $\Delta \chi^{2}=16.08, d f=30, p>.98$. Finally, we note that all of the slope variances were nonsignificant and the variances in the old age groups tend to be somewhat reduced as compared to the total sample. However, feeding the model with estimates from the total sample did not reduce the fit as compared to a model freely estimating the slope variances, $\Delta \chi^{2}=25.61, d f=$ $16, p>.06$.

In summary, the pattern of estimates for the sample that did not include impending death or dementia during the study was similar to those from the total sample.

\section{Discussion}

The two main results of this study are that correlations among levels of performance in different cognitive functions increase in, but not before, old age (over 65 years), and that interindividual differences in change could only be detected in old age. The results are more consistent with dynamic dedifferentiation (i.e., progressively increasing correlations among cognitive changes) than with stationary dedifferentiation (constant increases of associations among cognitive changes) over the adult lifespan.

Dynamic dedifferentiation in late life would be supported by age-related (a) increases in the correlations between levels of performance on cognitive abilities, (b) by increases in correlations among cognitive changes, and (c) by higher interindividual variability in change in the older age groups. Age-related increases in variances in change support the dynamic dedifferentiation hypothesis because between-subject differences in change are a necessary condition for associations among cognitive changes and increases in correlations among levels of cognitive functioning. That is, if rearrangement of rank ordering does not occur over time or across age groups, then no changes in correlations can be observed. In this study, interindividual differences in rates of cognitive aging begin to increase from middle adulthood onward for recall and from early old age to later old age for fluency and knowledge. The estimates of interindividual variances in change among older age groups contrast with the patterns of consistently very low and non-significant estimates for the three younger groups. Thus, very little rearrangement of rank ordering of individuals occurs in younger adulthood. Therefore, correlations among cognitive performance levels cannot change much. Note, however, that recent simulation work with LGM (Hertzog, Lindenberger, Ghisletta, \& von Oertzen, 2006) suggests that the power to detect variances in change is surprisingly low with the typical data sets 
from longitudinal studies on cognitive aging that contain relatively few assessments and moderately reliable measures. Thus, we are reluctant to endorse the null hypothesis of no interindividual differences in change in the younger age groups. Future research needs to address the amount of interindividual differences in change throughout the adult lifespan using more intensive, repeated measures designs.

Given the few significant variances in change, it was not possible to estimate their covariances. That is, the covariances among the changes (i.e., slopes) were not empirically identified because there were no variances in the slopes (i.e., they were constant over individuals). However, we could indirectly address the degree to which changes in the four cognitive measures were associated by evaluating the age-related pattern of increases in correlations among levels of performance; that is, if changes in different abilities are related, correlations between levels of functioning in different cognitive abilities should increase as a function of age. Specifically, rank ordering of individuals is more strongly determined by aging-related variance in samples of older adults than in samples of younger adults. Consequently, if cognitive changes in different abilities are correlated, correlations among levels of functioning in different abilities should increase as a function of age (Hofer \& Sliwinski, 2001). The results revealed a clear pattern of dedifferentiation with the median correlation among performance levels increasing from .52 in the youngest group to .74 in the oldest group. For four of the six individual correlations, age-group differences were significant. Thus, this study adds credibility to previous studies reporting increments in correlations among cognitive functions (e.g., Baltes \& Lindenberger, 1997; Hultsch et al., 1998; Li et al., 2004) and extends these studies by revealing a pattern of dedifferentiation across sequential narrow age cohorts, thereby removing the possibility of mean age trends to bias the correlations in a positive direction.

Several studies have reported significant amounts of interindividual variances in cognitive change in samples of older adults (e.g., Anstey et al., 2003b; Hultsch et al., 1998; Lövdén et al., 2004; Wilson et al., 2002). Conceptually, variances in change observed in longitudinal panel studies reflect at least two forms of heterogeneity: (a) heterogeneity in sample composition, or the presence of qualitatively separable populations of aging individuals within the same sample; and (b) heterogeneity in the timing of normative (e.g., population-general) change. For methodological and conceptual reasons, the demarcation lines between these two forms of heterogeneity tend to be blurred. Generally, dementia (e.g., Sliwinski et al., 2003) is said to contribute to sample heterogeneity (but see Drachman, 1994), whereas between-person differences in change related to deficiencies such as prefrontal functioning and dopaminergic neuromodulation (see Buckner, 2004) may predominantly point to heterogeneity in timing (i.e., these changes are assumed to eventually afflict most members of the aging population, but to varying degrees and at varying ontogenetic time points). In the present study, significant interindividual differences in change were only noted when individuals developing dementia or dying within the tenyear course of the study were included in the analyses. Moreover, the numerical patterns of findings suggested reduction in variances in change when these individuals were removed. However, the estimates from the reduced sample did not differ significantly from the estimates from the total sample, suggesting that the dementia- and mortality-related cognitive changes are not the only sources of dedifferentiation. Future research needs to further examine the extent to which the patterns of covariance dedifferentiation and increases in betweenperson variances are due to heterogeneity in forms of aging (e.g., dementia versus normal aging) or to heterogeneity in the timing of population-general processes. Moreover, the extent to which dedifferentiation generalizes from the between-subject level to the intra-individual level is largely unknown.

To conclude, we examined the extent of interindividual differences in cognitive change and the extent to which changes in different cognitive functions covary. To this end, we applied multivariate multi-cohort LGM to data from sequential narrow cohorts. The results revealed higher correlations among performance levels in different cognitive measures in, but not before, old age, continuation of age-related increases of correlations among cognitive performance levels in old age, and greater interindividual differences in change in older age groups than in younger age groups. Taken together, and in line with original formulations of the dedifferentiation hypothesis (for review, see Baltes et al., 2006), our results support a dynamic, rather than stationary, view of cognitive aging.

\section{Acknowledgement}

The Betula Longitudinal Study is funded by the Bank of Sweden Tercentenary Foundation (Grant 19880082:17), the Swedish Council for Planning and Coordination of Research (Grants D1988-0092, D1989-0115, D1990-0074, D1991-0258, D1992-0143, D1997-0756, D1997-1841, D1999-0739, and B1999-474), the Swedish Council for Research in the Humanities and Social 
Sciences (Grant F377/1988-2000), and the Swedish Council for Social Research (1998-1990: Grants 880082 and 311/1991-2000). Cindy de Frias is supported by a postdoctoral fellowship from the Canadian Institutes of Health Research. Work on this article was conducted when the first author held a postdoctoral position at the Department of Psychology at Stockholm University, Sweden. We acknowledge the contribution by the staff in the Betula project and thank Florian Schmiedek for numerous productive discussions.

\section{References}

Allen, P. A., Hall, R. J., Druley, J. A., Smith, A. F., Sanders, R. E., \& Murphy, M. D. (2001). How shared are age-related influences on cognitive and noncognitive variables? Psychology and Aging, 16, 532-549.

Anstey, K. J., Hofer, S. M., \& Luszcz, M. A. (2003a). Cross-sectional and longitudinal patterns of dedifferentiation in late-life cognitive and sensory function: The effects of age, ability, attrition, and occasion of measurement. Journal of Experimental Psychology. General, 132, 470-487.

Anstey, K. J., Hofer, S. M., \& Luszcz, M. A. (2003b). A latent growth curve analysis of late-life sensory and cognitive function over 8 years: Evidence for specific and common factors underlying change. Psychology and Aging, 18, 714-726.

Arbuckle, J. L. (1996). Full information estimation in the presence of incomplete data. In G. A. Marcoulides \& R.E. Schumacker (Eds.), Advanced structural equation modeling: Issues and techniques (pp. 243-277). Mahwah, NJ: Erlbaum.

Arbuckle, J. L., \& Wothke, W. (1999). Amos 4.0 user's guide. Chicago: Smallwaters.

Balinsky, B. (1941). An analysis of the mental factors of various age groups from nine to sixty. Genetic Psychology Monographs, 23, 191-234.

Baltes, P. B., \& Lindenberger, U. (1997). Emergence of a powerful connection between sensory and cognitive functions across the adult life span: A new window to the study of cognitive aging? Psychology and Aging, 12, 12-21.

Baltes, P. B., Lindenberger, U., \& Staudinger, U. M. (2006). Life span theory in developmental psychology. In W. Damon \& R.M. Lerner (Eds.), Handbook of child psychology, 6th ed. Theoretical models of human development, Vol. 1 (pp. 569-664) New York: Wiley.

Buckner, R. L. (2004). Memory and executive function in aging and $\mathrm{AD}$ : Multiple factors that cause decline and reserve factors that compensate. Neuron, 44, 195-208.

Dillon, W. R., Kumar, A., \& Mulani, N. (1987). Offending estimates in covariance structureanalysis: Comments on the causes of and solutions to Heywood cases. Psychological Bulletin, 101, 126-135.

Drachman, D. A. (1994). If we live long enough, will we all be demented? Neurology, 44, 1563-1565.

Dureman, I. (1960). SRB:1. Stockholm: Psykologiförlaget.

Ghisletta, P., \& Lindenberger, U. (2003). Age-based structural dynamics between perceptual speed and knowledge in the Berlin Aging Study: Direct evidence for ability dedifferentiation in old age. Psychology and Aging, 18, 696-713.

Hertzog, C., Dixon, R. A., Hultsch, D. F., \& MacDonald, S. W. S. (2003). Latent change models of adult cognition: Are changes in processing speed and working memory associated with changes in episodic memory? Psychology and Aging, 18, 755-769.

Hertzog, C., Lindenberger, U., Ghisletta, P., \& von Oertzen, T. (2006). On the power of multivariate latent growth curve models to detect correlated change. Psychological Methods, 11, 244-252.

Hofer, S. M., \& Sliwinski, M. J. (2001). Understanding ageing: An evaluation of research designs for assessing the interdependence of ageing-related changes. Gerontology, 47, 341-352.

Hofer, S. M., Sliwinski, M. J., \& Flaherty, B. P. (2002). Understanding ageing: Further commentary on the limitations of cross-sectional designs for ageing research. Gerontology, 48, 22-29.

Hultsch, D. F., Hertzog, C., Dixon, R. A., \& Small, B. J. (1998). Memory change in the aged. New York: Cambridge University Press.

Kline, R. B. (1998). Principles and practice of structural equation modeling. New York: Guilford Press.

Li, S. -C., Lindenberger, U., Hommel, B., Aschersleben, G., Prinz, W., \& Baltes, P. B. (2004). Lifespan transformations in the couplings of mental abilities and underlying cognitive processes. Psychological Science, 15, 155-163.

Li, S. -C., \& Schmiedek, F. (2002). Aged is not aging: Understanding the clocks that time the aging mind. Gerontology, 48, 5-12.

Lindenberger, U., \& Baltes, P. B. (1994). Sensory functioning and intelligence in old age: A strong connection. Psychology and Aging, 9, 339-355.

Lövdén, M., Bergman, L., Adolfsson, R., Lindenberger, U., \& Nilsson, L. -G. (2005). Studying individual aging in an interindividual context: Typical paths of age-related, dementiarelated, and mortality-related cognitive development in old age. Psychology and Aging, 20, 303-316.

Lövdén, M., \& Lindenberger, U. (2005). Development of intellectual abilities in old age: From age gradients to individuals. In $\mathrm{O}$. Wilhelm \& R. Engle (Eds.), Understanding and measuring intelligence (pp. 203-221). Thousand Oaks, CA: Sage.

Lövdén, M., Rönnlund, M., Wahlin, Å., Bäckman, L., Nyberg, L., \& Nilsson, L. -G. (2004). The extent of stability and change in episodic and semantic memory in old age: Demographic predictors of level and change. Journals of Gerontology. Psychological Sciences, 59B, 130-134.

McArdle, J. J., \& Epstein, D. (1987). Latent growth curves within developmental structural equation models. Child Development, 58, 110-133.

Meredith, W., \& Tisak, J. (1990). Latent growth curve analysis. Psychometrika, 55, 117-122.

Nilsson, L. G., Adolfsson, R., Bäckman, L., de Frias, C. M., Molander, B., \& Nyberg, L. (2004). Betula: A prospective cohort study on memory, health and aging. Aging, Neuropsychology, and Cognition, 11, 134-148.

Nilsson, L. -G., Bäckman, L., Erngrund, K., Nyberg, L., Adolfsson, R., Bucht, G., et al. (1997). The Betula prospective cohort study: Memory, health, and aging. Aging, Neuropsychology, and Cognition, 4, 1-32.

Nyberg, L., Maitland, S. B., Rönnlund, M., Bäckman, L., Dixon, R. A., Wahlin, A., et al. (2003). Selective adult age differences in an ageinvariant multifactor model of declarative memory. Psychology and Aging, 18, 149-160.

Rabbitt, P. (1993). Does it all go together when it goes? The Nineteenth Bartlett Memorial Lecture. Quarterly Journal of Experimental Psychology, 46, 385-434.

Raudenbush, S. W., \& Bryk, A. S. (2002). Hierarchical linear models: Applications and data analysis methods (2nd ed.). Thousand Oaks, CA: Sage.

Raz, N., Lindenberger, U., Rodrigue, K. M., Kennedy, K. M., Head, D., Williamson, A., et al. (2005). Regional brain changes in aging 
healthy adults: General trends, individual differences and modifiers. Cerebral Cortex, 15, 1676-1689.

Rönnlund, M., Nyberg, L., Bäckman, L., \& Nilsson, L. -G. (2005). Stability, growth, and decline in adult life span development of declarative memory: Cross-sectional and longitudinal data from a population-based study. Psychology and Aging, 20, 3-18.

Rubin, D. B. (1974). Estimating causal effects of treatments in randomized and nonrandomized studies. Journal of Educational Psychology, 66, 688-701.

Schafer, J. L., \& Graham, J. W. (2002). Missing data: Our view of the state of the art. Psychological Methods, 7, 147-177.

Schaie, K. W., Maitland, S. B., Willis, S. L., \& Intrieri, R. C. (1998). Longitudinal invariance of adult psychometric ability factor structures across 7 years. Psychology and Aging, 13, 8-20.

Singer, T., Verhaeghen, P., Ghisletta, P., Lindenberger, U., \& Baltes, P. B. (2003). The fate of cognition in very old age (BASE): Six-year longitudinal findings and methodological implications of age-associated selectivity. Psychology and Aging, 18, 318-331.
Sliwinski, M. J., Hofer, S. M., \& Hall, C. (2003). Correlated and coupled cognitive change in older adults with and without preclinical dementia. Psychology and Aging, 18, 672-683.

Wechsler, D. (1991). Manual for the Wechsler adult intelligence scale-revised. New York: The Psychological Corporation.

Wilson, R. S., Beckett, L. A., Barnes, L. L., Schneider, J. A., Bach, J., Evans, D. A., et al. (2002). Individual differences in rates of change in cognitive abilities of older persons. Psychology and Aging, 17, 179-193.

Wothke, W. (2000) Longitudinal and multi-group modeling with missing data. In T.D. Little, K.U. Schnabel, J. Baumert (Eds.) Modeling longitudinal and multilevel data: Practical issues, applied approaches and specific examples (pp. 219-240, 269-281). Mahwah, NJ: Erlbaum.

Zelinski, E. M., \& Lewis, K. L. (2003). Adult age differences in multiple cognitive functions: Differentiation, dedifferentiation, or process-specific change? Psychology and Aging, 18, 727-745.

Zelinski, E. M., \& Stewart, S. (1998). Individual differences in 16year memory changes. Psychology and Aging, 13, 622-630. 